\title{
Using Mobile Health Tools to Assess Physical Activity Guideline Adherence and Smoking Urges: Secondary Analysis of mActive-Smoke
}

Rongzi Shan ${ }^{1,2}$, BSc; Lisa R Yanek ${ }^{3}$, MPH; Luke G Silverman-Lloyd ${ }^{1,4}$, BA; Sina Kianoush ${ }^{1,5}$, MD, MPH; Michael J Blaha $^{1}$, MD, MPH; Charles A German ${ }^{6}$, MD; Garth N Graham ${ }^{7}$, MD, MPH; Seth S Martin ${ }^{1}$, MD, MHS

\footnotetext{
${ }^{1}$ Ciccarone Center for the Prevention of Cardiovascular Disease, Division of Cardiology, Department of Medicine, Johns Hopkins University School of Medicine, Baltimore, MD, United States

${ }^{2}$ David Geffen School of Medicine at University of California Los Angeles, Los Angeles, CA, United States

${ }^{3}$ Department of Medicine, Johns Hopkins University School of Medicine, Baltimore, MD, United States

${ }^{4}$ University of California, Berkeley-University of California, San Francisco Joint Medical Program, Berkeley, CA, United States

${ }^{5}$ Yale New Haven Medical Center - Waterbury Hospital, Waterbury, CT, United States

${ }^{6}$ Heart and Vascular Center of Excellence, Wake Forest Baptist Health, Winston-Salem, NC, United States

${ }^{7}$ Aetna Foundation, Hartford, CT, United States
}

\section{Corresponding Author:}

Seth S Martin, MD, MHS

Ciccarone Center for the Prevention of Cardiovascular Disease

Division of Cardiology, Department of Medicine

Johns Hopkins University School of Medicine

$600 \mathrm{~N}$ Wolfe Street

Carnegie 591

Baltimore, MD, 21287

United States

Phone: 14105020469

Email: $\underline{\text { smart100@jhmi.edu }}$

\section{Abstract}

Background: Rates of cigarette smoking are decreasing because of public health initiatives, pharmacological aids, and clinician focus on smoking cessation. However, a sedentary lifestyle increases cardiovascular risk, and therefore, inactive smokers have a particularly enhanced risk of cardiovascular disease.

Objective: In this secondary analysis of mActive-Smoke, a 12-week observational study, we investigated adherence to guideline-recommended moderate-to-vigorous physical activity (MVPA) in smokers and its association with the urge to smoke.

Methods: We enrolled 60 active smokers ( $\geq 3$ cigarettes per day) and recorded continuous step counts with the Fitbit Charge HR. MVPA was defined as a cadence of greater than or equal to 100 steps per minute. Participants were prompted to report instantaneous smoking urges via text message 3 times a day on a Likert scale from 1 to 9 . We used a mixed effects linear model for repeated measures, controlling for demographics and baseline activity level, to investigate the association between MVPA and urge.

Results: A total of 53 participants (mean age 40 [SD 12] years, 57\% [30/53] women, 49\% [26/53] nonwhite, and 38\% [20/53] obese) recorded 6 to 12 weeks of data. Data from 3633 person-days were analyzed, with a mean of 69 days per participant. Among all participants, median daily MVPA was 6 min (IQR 2-13), which differed by sex (12 min [IQR 3-20] for men vs 3.5 min [IQR 1-9] for women; $P=.004)$ and BMI (2.5 min [IQR 1-8.3] for obese vs $10 \mathrm{~min}$ [IQR 3-15] for nonobese; $P=.04)$. The median total MVPA minutes per week was 80 (IQR 31-162). Only $10 \%$ (5/51; 95\% CI 4\% to 22\%) of participants met national guidelines of 150 min per week of MVPA on at least $50 \%$ of weeks. Adjusted models showed no association between the number of MVPA minutes per day and mean daily smoking urge $(P=.72)$.

Conclusions: The prevalence of MVPA was low in adult smokers who rarely met national guidelines for MVPA. Given the poor physical activity attainment in smokers, more work is required to enhance physical activity in this population. 
(JMIR Cardio 2020;4(1):e14963) doi: 10.2196/14963

\section{KEYWORDS}

physical activity; smoking; mHealth; fitness trackers; short message service

\section{Introduction}

\section{Background}

Smoking cessation and physical activity both lead to significant improvements in health [1]. Although smoking rates are decreasing because of regulation and taxation, behavioral counseling, and pharmacotherapy [1], an individual's attempts to quit smoking are still challenging [2]. Furthermore, because a sedentary lifestyle increases cardiovascular risk [3], inactive smokers have a particularly enhanced risk of cardiovascular disease. The 2018 US Physical Activity Guidelines recommend greater than or equal to 150 min per week moderate activity or greater than or equal to 75 min per week vigorous physical activity (VPA), accumulated over bouts of any duration [4]. However, the prevalence of meeting these activity guidelines in the general adult population is unsatisfactory, with half of the US adults attaining fewer than $150 \mathrm{~min}$ of moderate-to-vigorous physical activity (MVPA) during leisure time per week, by self-report [4]. Moreover, 2 studies in young adults [5] and youth aged 14 to 18 years [6] found that self-reported attainment of physical activity guidelines was positively associated with noncigarette forms of tobacco use (eg, electronic cigarettes) but inversely associated with cigarette smoking, suggesting that physical inactivity and cigarette smoking may be compounding risk factors.

Physical activity has been suggested as an aid for smoking cessation, potentially through moderation of cravings and prevention of weight gain $[7,8]$, but evidence is conflicting. Although there is insufficient evidence to recommend exercise as an aid for smoking cessation [7,9], previous meta-analyses suggested that acute bouts of exercise decrease urges, with activities at moderate to vigorous intensity having the greatest effect $[10,11]$. In addition, a study on active smokers found that a higher level of habitual MVPA was significantly associated with lower smoking urges [12]. However, this study used 7-day physical activity recall for assessing the levels of MVPA, leading to potential recall bias.

Methodological limitations such as recall bias and poor ecological validity are common in prior studies of exercise and smoking. Self-report for physical activity has been shown to poorly correlate with accelerometer data in accurately measuring MVPA and sedentary time, whereas ecological momentary assessment using mobile health (mHealth) tools, designed to sample real-time behaviors and experiences in the natural environment, performed better [13].

\section{Objectives}

The goal of mActive-Smoke, a 12-week prospective observational study, was to assess the day-level association between objectively measured physical activity and concurrent smoking urges. Previously reported primary results [14] demonstrate that acute bouts of physical activity (ie, number of steps accumulated in a 30-min period before urge reporting), but not total daily steps, were associated with a modest decrease in smoking urges. Although we previously found a temporal association between acute bouts of activity and decreased urge, it is unclear whether the intensity of daily activity is associated with daily urge. In addition, despite well-established contributions of physical inactivity [3] and smoking on cardiovascular risk, there is limited research describing adherence to physical activity guidelines in adult smokers, with prior research mostly reliant on self-reported data. Thus, in this secondary analysis, we used prospective, objective measures to investigate adherence to guideline-recommended MVPA and the association between the intensity of physical activity and the urge to smoke.

\section{Methods}

\section{Study Aim and Design}

The aims of this secondary analysis were to report adherence to physical activity guidelines among smokers in the mActive-Smoke study population and to investigate the relationship between the intensity of physical activity and the urge to smoke. The methods for this 12-week prospective observational study have been previously reported [14], and a summary is provided below (Recruitment and Measurement of Baseline Variables and Data Collection). This study was approved by the Johns Hopkins School of Medicine Institutional Review Board.

\section{Recruitment and Measurement of Baseline Variables}

We recruited 60 participants from April 7, 2016, to September 2, 2016, using on-site advertisements, social media, and physician referrals. Participants met inclusion criteria if they were aged 18 years or older, smoked at least 3 cigarettes per day on average, owned a smartphone, and were able to perform normal physical activity. Participants were screened for eligibility via email. At an initial meeting with a study coordinator, participants completed an enrollment questionnaire to record demographic characteristics, self-reported BMI (weight $[\mathrm{kg}] /$ height $\left[\mathrm{m}^{2}\right]$ ), physical activity, and smoking behavior. Baseline physical activity was assessed by the International Physical Activity Questionnaire (IPAQ)-short form, a questionnaire assessing walking time, sedentary time, and MVPA time in the past 7 days [15]. A high IPAQ score is defined as the equivalent of either VPA on 3 days or more per week at greater than or equal to 1500 metabolic equivalent of task (MET) minutes per week or 5 days or more per week of any combination of MVPA meeting greater than or equal to 3000 MET minutes per week [16]. Baseline smoking behavior was assessed with the Arizona Smoking Assessment Questionnaire [17].

\section{Data Collection}

For the measurement of physical activity, participants used the Fitbit Charge HR (Fitbit Inc), a wrist-worn triaxial digital 
accelerometer allowing continuous monitoring of activity and heart rate. Patients were not instructed to alter their physical activity, but they could access step counts via the Fitbit mobile app (Fitbit Inc). Data from the Fitbit, including steps and the Fitbit-generated intensity level, were compiled in Fitabase, a secure research platform that collects real-time data from activity tracking devices [18]. Day-level and minute-level data were downloaded from Fitabase for each participant.

To measure smoking urges, an automated messaging service sent SMS text messages to participants 3 times per day, requesting that they respond with their instantaneous urge to smoke on a 9-point Likert scale from low to high. These messages were sent at participant-defined times, corresponding roughly to waking up, lunchtime, and returning home at the end of a day.

Participants were asked to complete a Web-based end-of-study survey regarding the study experience and their perceptions on physical activity and smoking urges. Survey questions and results were previously reported [14].

\section{Statistical Analysis}

Baseline characteristics were summarized using descriptive statistics, frequency for categorical data and mean (SD) and median (IQR) for continuous data. Spearman and Pearson correlation coefficients were used for associations between variables.

As participants were not required to wear Fitbits during sleep, we defined nonwear time as 90 consecutive minutes of missing heart rate data between the hours of $10 \mathrm{am}$ and $10 \mathrm{pm}$. Days with 2 or more 90 -min nonwear periods and wear time of fewer than 6 hours within the target time window were excluded [19]. At least 6 total weeks of recorded data were required for inclusion. For the calculation of the prevalence of meeting weekly MVPA goals, we included weeks for which participants contributed 4 or more days of complete data [20].

Fitbit assigns minute-level activity into 4 intensity levels (0: sedentary, 1: light, 2: moderate, and 3: vigorous) [18]. We eschewed this measure of intensity given the proprietary algorithms and concern about accuracy $[21,22]$ and used cadence as a surrogate measure of intensity. We elected to not include heart rate because of concerns about the accuracy of Fitbit's heart rate measurement, particularly at vigorous intensities [23]. However, to explore the nature of Fitbit's intensity variable, we compare daily MVPA minutes by Fitbit intensity levels (number of minutes spent at intensity level 2 or 3) with daily MVPA minutes by cadence threshold.

Cadence (steps per minute) is associated with objectively measured speed and intensity under controlled experimental conditions [24]. A threshold of 100 steps per minute is an evidence-based value generally associated with moderate intensity or greater than or equal to 3 METs and is best described as brisk walking, whereas a threshold of 130 steps per minute is associated with vigorous intensity or greater than or equal to 6 METs [25]. We created 4 cadence categories defined as 0 steps per minute (no movement), 1 to 59 steps per minute (incidental movement to purposeful steps), 60 to 99 steps per minute (slow to medium walking), and greater than or equal to
100 steps per minute (brisk walking and faster), whereas VPA was defined as cadence of greater than or equal to 130 steps per minute [25]. Daily minutes of MVPA were calculated by summing the minutes spent at cadence of greater than or equal to 100 steps per minute, and daily minutes of VPA was calculated by summing the minutes spent at cadence of greater than or equal to 130 steps per minute. We also calculated moderate $+2 \times \mathrm{VPA}$, which weights moderate activity as $1 \mathrm{~min}$ and vigorous activity as $2 \mathrm{~min}$, in accordance with physical activity guidelines [20], but as the results remained similar, we reported only MVPA and VPA.

Given the positively skewed data, we described MVPA minutes using median (IQR) and used the Wilcoxon rank sum test for comparing between-group differences. We reported the within-person and between-person prevalence of physical activity guideline adherence. We also estimated the prevalence of adherence with obtaining at least 150 min of MVPA or 75 min of VPA per week on greater than or equal to $50 \%$ of the study weeks. Although the physical activity guidelines were developed based on self-report, we opted to apply the physical activity guidelines to cadence measurements to provide a clinical context for the objective data. Daily urge to smoke was described using mean (SD) of the 3 to 4 urge messages sent each day. The mean daily urge was normally distributed and was treated as a continuous variable.

A repeated measures multivariable mixed effects linear model, accounting for autoregression and heteroscedasticity, was used to evaluate the relationship between daily MVPA minutes and daily urge. We adjusted for age, sex, race, education, BMI, baseline cigarettes per day, and baseline physical activity, which were selected a priori. Baseline physical activity was defined as a high level of activity or not by IPAQ. We explored interactions between age, sex, obesity status, baseline physical activity, and baseline cigarettes per day with daily MVPA minutes, with $P<.10$ considered evidence of interaction. The analysis was conducted using Stata (version 15.1; StataCorp).

\section{Results}

\section{Study Flow and Baseline Characteristics}

The study flow diagram, baseline characteristics, and survey results have been previously reported [14]. In brief, 60 participants were enrolled, and 53 participants recorded at least 6 weeks of data and were thus included in this analysis. In addition, 45 participants recorded 12 weeks of data, and 8 participants recorded 6 to 12 weeks of data. Of all participant-weeks, $80.1 \%$ (144/723) of weeks included at least 4 days of complete data. Participants sent a mean of 290 (SD 62) SMS text messages quantifying the urge to smoke. Moreover, 49 participants completed the Web-based exit survey. After excluding days using nonwear criteria, data from 3633 days were analyzed, with a mean of 69 days of data contributed by each participant. The mean age was 40 (SD 12) years, with $57 \%$ (30/53) women, $49 \%$ (26/53) nonwhite participants, and $30 \%(16 / 53)$ with a bachelor's degree or higher. In addition, $40 \%$ (21/53) of participants were overweight, 38\% (20/53) were obese, and $53 \%(28 / 53)$ had a high level of baseline activity as assessed by IPAQ (Table 1). 
Table 1. Baseline characteristics of mActive-Smoke participants $(\mathrm{N}=53)$.

\begin{tabular}{|c|c|}
\hline Characteristic & Participants, n (\%) \\
\hline \multicolumn{2}{|l|}{ Sex } \\
\hline Men & $23(43)$ \\
\hline Women & $30(57)$ \\
\hline \multicolumn{2}{|l|}{ Age (years) } \\
\hline $22-29$ & $11(21)$ \\
\hline $30-39$ & $16(30)$ \\
\hline $40-49$ & $15(28)$ \\
\hline $50-59$ & $7(13)$ \\
\hline $60-65$ & $4(8)$ \\
\hline \multicolumn{2}{|l|}{ Race } \\
\hline White & $27(51)$ \\
\hline Nonwhite & $26(49)$ \\
\hline \multicolumn{2}{|l|}{ Education } \\
\hline High school diploma or less & $8(15)$ \\
\hline Associate degree or some college & $29(55)$ \\
\hline Bachelor's degree or higher & $16(30)$ \\
\hline \multicolumn{2}{|l|}{ BMI $\left(\mathrm{kg} / \mathrm{m}^{2}\right)$} \\
\hline$<25.0$ (normal or underweight) & $12(23)$ \\
\hline 25.0-29.9 (overweight) & $21(40)$ \\
\hline$\geq 30.0$ (obese) & $20(38)$ \\
\hline \multicolumn{2}{|c|}{ International Physical Activity Questionnaire ${ }^{\mathrm{a}}$} \\
\hline Low & $6(11)$ \\
\hline Moderate & $19(36)$ \\
\hline High & $28(53)$ \\
\hline \multicolumn{2}{|l|}{ Cigarettes smoked per day } \\
\hline$\leq 10$ & $34(64)$ \\
\hline$>10$ & $19(36)$ \\
\hline
\end{tabular}

${ }^{\mathrm{a} C a t e g o r i e s ~ d e f i n e d ~ b y ~ I n t e r n a t i o n a l ~ P h y s i c a l ~ A c t i v i t y ~ Q u e s t i o n n a i r e ~ g u i d e l i n e s . ~}$

\section{Patterns of Physical Activity}

Participants accumulated a median of 7807 steps per day (IQR 5383-10,824). Of 53 participants, 31 (58\%) met the recommended 30 min per day of MVPA on 1 or more days over the study duration. Of these 31 participants, the 30 min per day MVPA goal was met on a mean of $19 \%$ of days. Prevalence of adherence to national physical activity guidelines, defined as the proportion of participants who obtained at least $150 \mathrm{~min}$ per week of MVPA on at least $50 \%$ of total weeks, was $10 \%$ (5/51; $95 \%$ CI $4 \%$ to $21 \%$ ). No participants attained at least $75 \mathrm{~min}$ per week of VPA on at least $50 \%$ of total weeks. Of the 53 participants, only 15 (28\%) met 150 min per week of MVPA at least once throughout the study, and those 15 participants met that goal on a mean of $36 \%$ of weeks.

Overall, the median total MVPA minutes per week was 80 (IQR 31-162). Among all participants, median daily MVPA (more than 100 steps per min) was 6 min (IQR 2-13), whereas median daily minutes spent in lighter activity (60-99 steps per min) was 23 min (IQR 17-24). The median number of minutes spent in MVPA was significantly higher among men (12 min, IQR 3-20) than women (3.5 min, IQR 1-9; $P=.004)$. The median number of minutes spent in lighter activity was also significantly higher among men (34 min, IQR 26-52) than women (18 min, IQR $15-23 ; \quad P<.001)$. When comparing obese and nonobese participants, only MVPA minutes were significantly different, with median of 10 min (IQR 3-15) in participants with BMI less than $30 \mathrm{~kg} / \mathrm{m}^{2}$ versus median of $2.5 \mathrm{~min}$ (IQR 1-8.3) in participants with BMI greater than or equal to $30 \mathrm{~kg} / \mathrm{m}^{2}(P=.04$; Table 2). There was poor correlation between the median daily MVPA minutes and baseline activity level (low, moderate, or high) as assessed by IPAQ (Spearman coefficient=-0.162; $P=.25)$. 
Table 2. Daily minutes spent at cadence bands by demographic characteristics over the study duration.

\begin{tabular}{|c|c|c|}
\hline Characteristic & 60 to 99 steps per minute, median (IQR) & More than 100 steps per minute, median (IQR) \\
\hline \multicolumn{3}{|l|}{ Sex } \\
\hline Men & $34(26-52)^{\mathrm{a}}$ & $12(3-20)^{\mathrm{a}}$ \\
\hline Women & $18(15-23)^{\mathrm{a}}$ & $3.5(1-9)^{\mathrm{a}}$ \\
\hline \multicolumn{3}{|l|}{ Age (years) } \\
\hline$<40$ & $28.5(17-44.3)$ & $6.25(2-15.5)$ \\
\hline$\geq 40$ & $22.5(18-29)$ & $5(2-12)$ \\
\hline \multicolumn{3}{|l|}{ BMI $\left(\mathrm{kg} / \mathrm{m}^{2}\right)$} \\
\hline$<30$ & $29(18-44.5)$ & $10(3-15)^{\mathrm{b}}$ \\
\hline$\geq 30$ & $21(15.5-28)$ & $2.5(1-8.3)^{\mathrm{b}}$ \\
\hline
\end{tabular}

${ }^{\mathrm{a}} P<.01$, two-sample Wilcoxon rank sum test.

${ }^{\mathrm{b}} P<.05$, two-sample Wilcoxon rank sum test.

\section{Association Between Day-Level Intensity and Urge}

The number of daily MVPA minutes was positively skewed, with no clear association with urge upon inspection (Figure 1). Furthermore, logarithmic transformation of the MVPA variable did not reveal any clear relation with mean daily urge. There was no significant association between daily MVPA minutes and mean daily urge to smoke in either the unadjusted model $(P=.74)$ or the adjusted model $(P=.72)$.
We explored the interaction of daily MVPA minutes with binary demographic factors, defined as age greater than or equal to 40 years, sex, BMI greater than or equal to $30 \mathrm{~kg} / \mathrm{m}^{2}$, and baseline high activity based on IPAQ. The $P$ values for interaction are as follows: .41 for age, .15 for sex, .90 for BMI, and .32 for high activity. Thus, no interaction terms were included in the adjusted models.

Figure 1. Mean daily smoking urge plotted against daily minutes of moderate-to-vigorous physical activity for all participants (N=53; 3633 person-days). MVPA: moderate-to-vigorous physical activity.

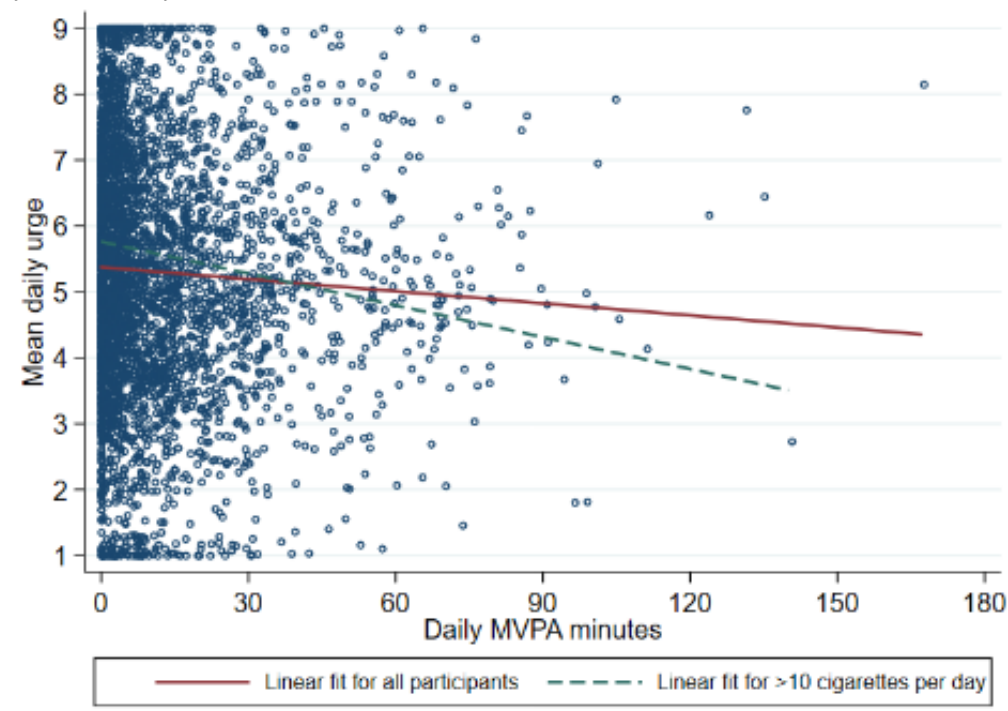

\section{Sensitivity Analysis}

Given that our prior work validating the urge to smoke revealed a positive association between mean urge over the course of the study and the number of cigarettes per day reported at the end of the study [14], we explored associations by baseline cigarette consumption. When stratifying by baseline cigarettes per day, those who smoked more than 10 cigarettes per day $(n=19 ; 1333$ person-days) had 0.293 lower daily urge per $30 \mathrm{~min}$ per day of MVPA ( $P=.03$; $95 \%$ CI -0.563 to -0.023 ; Figure 1$)$, but this was not significant on stratifying by greater than equal to 15
( $\mathrm{n}=16 ; 1100$ person-days; $P=.80$ ) or greater than equal to 20 cigarettes per day $(\mathrm{n}=11 ; 809$ person-days; $P=.88)$.

\section{Comparison of Cadence Versus Fitbit's Intensity Levels}

To elucidate the nature of Fitbit's intensity variable, we compared the distribution of daily MVPA minutes calculated using the definition of cadence greater than or equal to 100 steps per minute with the distribution of daily MVPA minutes as defined by the number of minutes spent at moderate- or vigorous-intensity levels as defined by Fitbit's algorithm (Figure 
2). Although there was some correlation between daily MVPA minutes by cadence and daily MVPA minutes by Fitbit intensity (Pearson coefficient $=0.58$ ), the minutes categorized by Fitbit as MVPA tended to have lower cadences than the threshold of 100 steps per minute. The minutes that Fitbit categorized as moderate intensity had a median of 41 steps accumulated within that minute (IQR 14-67, range 0-138), whereas minutes categorized as vigorous intensity had a median of 95 steps accumulated (IQR 57-106, range 0-215). This led to a wider spread of daily MVPA minutes by Fitbit intensity (range 0-451 min) than by cadence (range $0-167 \mathrm{~min}$ ).

Figure 2. Daily moderate-to-vigorous physical activity (MVPA) minutes calculated using cadence thresholds versus daily MVPA minutes calculated using Fitbit's intensity levels.

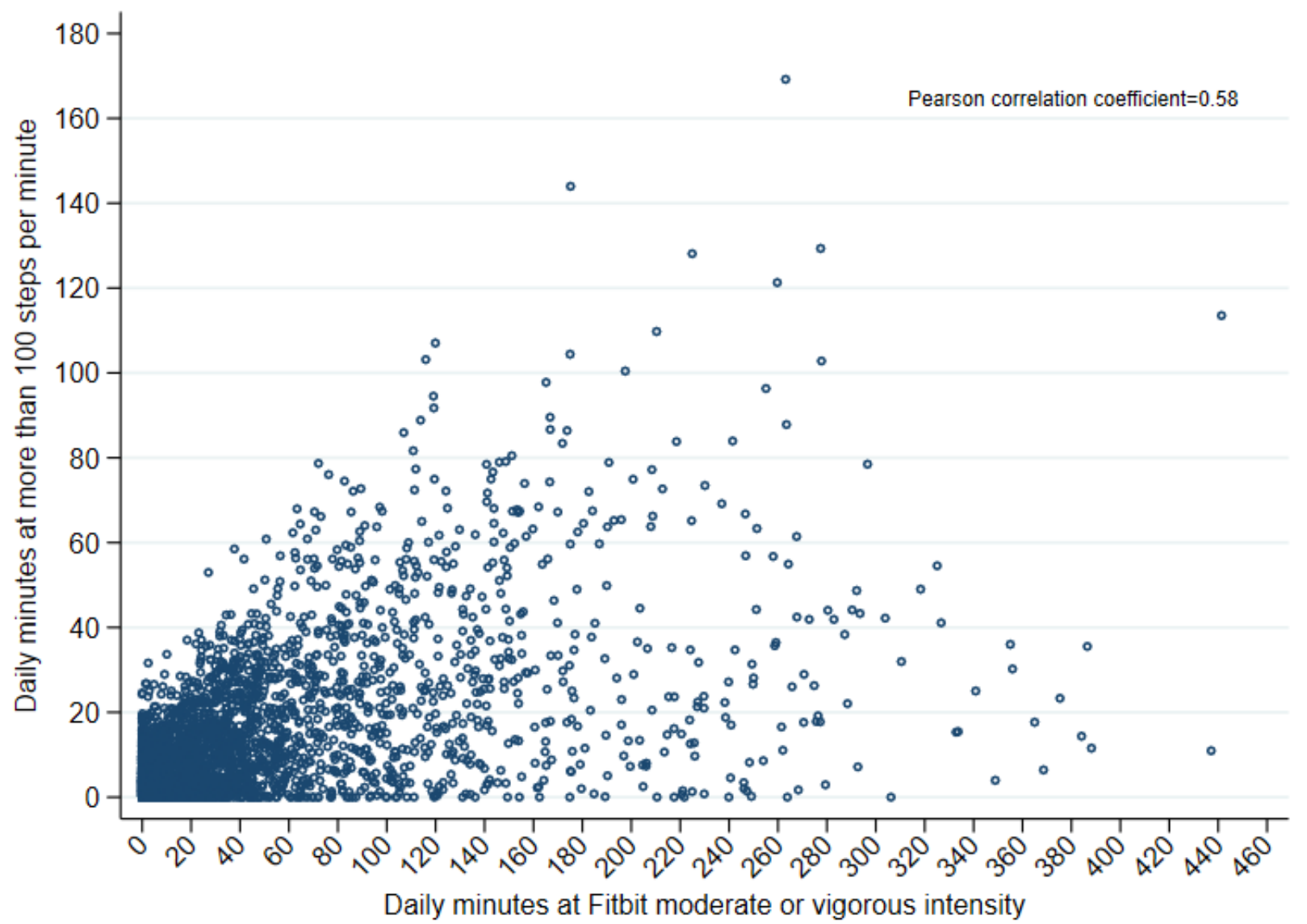

\section{Discussion}

\section{Principal Findings}

In this secondary analysis of data from mActive-Smoke, we described intensity of physical activity in free-living adult smokers and found that the prevalence of MVPA was low, with $10 \%(5 / 51)$ of participants attaining greater than or equal to 150 min of MVPA on at least $50 \%$ of study weeks and no participants attaining greater than or equal to $75 \mathrm{~min}$ of VPA on at least $50 \%$ of study weeks. Overall, the median daily MVPA was 6 min, and this differed by sex and BMI. Most participants achieved at least 30 min per day of light-intensity activity (60-99 steps per minute) over the study duration. In regression analyses, there was no association between daily MVPA minutes and mean daily smoking urges among all participants. In addition, this study provides exploratory insights on using Fitbit's intensity level to determine MVPA, compared with accepted cadence thresholds, which is a simpler marker of intensity available across measurement devices.

\section{Comparison With Prior Work}

This study highlights the low prevalence of MVPA in adult smokers in a free-living environment and poor adherence to the 2018 US Physical Activity Guidelines of greater than or equal to $150 \mathrm{~min}$ per week of MVPA. Our results corroborate observations by prior analyses of accelerometer data from the National Health and Nutrition Examination Survey (NHANES), which are representative of the general US population. Using 2005 to 2006 NHANES data, Tucker et al [20] found that $59.6 \%$ of adults met the 2008 US Physical Activity Guidelines by self-report, whereas $8.6 \%$ of adults met the guidelines by accelerometer measurement, using the goal of greater than or equal to $150 \mathrm{~min}$ per week of MVPA in the 10-min bout. In the 2018 US Physical Activity Guidelines, the 10-min bout requirement was removed; thus, we did not include bouts in the calculation of MVPA minutes. Doing so would likely further reduce the estimated attainment of recommended physical activity levels and, as such, would not impact the conclusion of low levels of physical activity guideline adherence. 
Another analysis of 2005 to 2006 NHANES data found that US adults accumulated only about 7 min per day of self-selected activity at a cadence of greater than or equal to 100 steps per minute (generally defined as MVPA), but the participants did accumulate, on average, about $30 \mathrm{~min}$ per day of activity at cadences of more than 60 steps per minute [26]. The NHANES dataset from these prior studies included accelerometer data over 1 to 7 days, whereas our data were obtained over 6 to 12 weeks $[20,26]$, thus suggesting that low levels of physical activity may persist over time. These results highlight a need for further work in promoting physical activity in smokers to mitigate the compounding cardiovascular risk factors of inactivity and smoking.

Although there was no intervention to increase physical activity, $82 \%(40 / 49)$ of participants reported in the exit survey that they believed the study helped increase their physical activity [14], affirming the potential of mHealth tools and self-monitoring in promoting behavioral change. Furthermore, the 2018 Physical Activity Guidelines recommend information technology and mHealth interventions as a future direction for tracking and promoting physical activity [4].

This study also addresses the recall bias in prior studies on physical activity and smoking urges. For example, Haasova et al [12] showed that more habitual MVPA minutes based on 7-day activity recall significantly correlated with decreased urge over the past 7 days in 98 smokers. The difference between results from this 7-day recall study and our analysis suggests that the prevalence of MVPA and granularity of data are important factors to consider in studies on physical activity and smoking urge. Specifically, median daily MVPA based on 7-day recall was 45 min (IQR 17-77) in the study by Hassova et al [12], whereas we measured median daily MVPA over 12 weeks to be 6 min (IQR 2-13) using minute-level and day-level granularity of data. This is unsurprising given that this analysis and prior studies [13] found poor correlation between self-reported intensity via IPAQ and accelerometer-measured intensity.

This study analyzed intensity on a day level by quantifying the total daily minutes of MVPA for each person-day, which builds on prior studies showing that short bouts of MVPA acutely decrease cigarette cravings in a controlled laboratory setting [11]. In addition, we build on our primary analysis of mActive-Smoke, which showed that increased rate of step accumulation within 15-, 30-, or 60-min time windows before urge reporting was associated with decreased urge. When comparing our results with these prior studies on acute effects, we conclude that although MVPA may modestly decrease the urge to smoke immediately after physical activity, the effect of MVPA on the urge to smoke does not appear to persist throughout the day.

\section{Limitations}

We acknowledge that this was a relatively small, single-center study, not generalizable to smokers everywhere. Despite the low prevalence of MVPA, the participants in this study had fairly high total daily step counts, suggesting that these participants may be active throughout the day at lighter intensities. This confers cardiovascular health benefits over a more sedentary lifestyle but may not be enough to affect smoking urges [4]. In addition, there is inherent selection bias, as participants who enrolled in this study were more likely to have an interest in behavior change, and step counts and urge reporting may be subject to the Hawthorne effect. However, it is important to note that even as part of a research study, the physical activity observed was low and similar to prior studies of the general population.

As this was a post hoc analysis, the sample size was not powered to test correlations between intensity and smoking urge. Although the total number of observations was high, the number of participants in this pilot study was relatively small, especially in the stratified models. However, we did account for repeated measures and autoregression in the model, and our smallest stratified group contained 809 person-days.

In addition, mActive-Smoke participants were lighter smokers, with 36\% (19/53) reporting more than 10 cigarettes per day at baseline. Prior studies generally used a minimum of 10 cigarettes per day as the threshold for study inclusion $[9,12]$. We did not collect data on the time since the last cigarette to avoid overburdening participants with text messages; thus, we could not adjust for the potential confounding effect of recent smoking on urge. These factors may have generated a flooring effect, as physical activity may be less able to further reduce smoking urge when the urge is already low, either from a recent cigarette or because of lighter smokers having lower urges. We previously validated the urge scale and found that self-reported urges correlated well with daily cigarette consumption.

Cadence is an imprecise marker of intensity, correlating well with caloric expenditure, but does not account for types of activity other than walking or running, leading to possible underestimation of MVPA. In addition, the cadence thresholds used in these analyses were not adjusted for stride length variation among participants. Bias from lack of stride length adjustment is likely to be minimal, as overestimation of MVPA in participants with shorter stride is partially offset by underestimation of MVPA in participants with longer stride. Although the Fitbit Charge HR reports minute-level intensity levels, METs, and heart rate, we opted for cadence as the measure of intensity, as it is less dependent on other factors such as resting heart rate, comorbidities, and medications. Furthermore, validation studies have raised concerns about the accuracy of Fitbit's reporting of heart rate [23], intensity, and energy expenditure, although step count was generally accurate [21,27].

Despite the advantages of objective activity measures, it is important to note that physical activity guidelines were developed based on self-reported data, and there are currently no guidelines based on accelerometer data. Linking objective measures with physical activity guideline attainment to provide clinical context has been done previously [20], but this method requires further validation in future studies. More work is needed to develop guidelines based on objective metrics of physical activity. Future directions include devising the optimal method of incorporating heart rate data into measurement of MVPA while accounting for medications and clinical characteristics. Finally, Fitbit provides other information about health behaviors, 
such as sleep, which could impact both physical activity and smoking urge and warrants further exploration.

\section{Conclusions}

In this 12-week observational study of adult smokers using mHealth tools for real-time assessment of physical activity and smoking urge, the prevalence of MVPA was low, and participants rarely met national guidelines for physical activity. We found no day-averaged association between intensity of activity and smoking urges. On the basis of the known benefits of physical activity and the low levels observed in this study, more work is needed to address physical activity promotion in smokers.

\section{Acknowledgments}

The Aetna Foundation funded the study through a grant to Johns Hopkins University and had no role in the study design, data collection, or statistical analysis. One writing team member from the Aetna Foundation participated in manuscript editing. The corresponding author had full access to all data and final responsibility for the decision to submit for publication.

\section{Authors' Contributions}

RS drafted the manuscript. RS, LRY, and LGSL analyzed the data. SK and LGSL collected the data. MJB, SSM, SK, and LGSL designed the study and developed the protocol. All authors edited the manuscript and approved the final version.

\section{Conflicts of Interest}

RS and LGSL report grants from the Aetna Foundation during the conduct of the study. LRY, SK, CAG, and GNG have nothing to disclose. MJB reports grants from the Aetna Foundation during the conduct of the study, grants from the National Institutes of Health, grants and personal fees from the US Food and Drug Administration, grants from the American Heart Association, grants from the American College of Cardiology, grants and personal fees from Amgen, personal fees from Novartis, personal fees from Sanofi, personal fees from Novo Nordisk, personal fees from Bayer, and personal fees from Medicure, outside the submitted work. SSM reports grants from the Aetna Foundation during the conduct of the study; personal fees from Amgen, Sanofi, Regeneron, Esperion, Novo Nordisk, Quest Diagnostics, and Akcea Therapeutics; and research support from Apple, Google, iHealth, Nokia, the Maryland Innovation Initiative, American Heart Association, PJ Schafer Memorial Fund, and David and June Trone Family Foundation, outside the submitted work. In addition, SSM has a pending patent on a System of low-density lipoprotein cholesterol estimation.

\section{References}

1. Benjamin EJ, Virani SS, Callaway CW, Chamberlain AM, Chang AR, Cheng S, American Heart Association Council on Epidemiology and Prevention Statistics Committee and Stroke Statistics Subcommittee. Heart disease and stroke statistics-2018 update: a report from the American Heart Association. Circulation 2018 Mar 20;137(12):e67-492. [doi: 10.1161/CIR.0000000000000558] [Medline: 29386200]

2. Hughes J, Keely J, Naud S. Shape of the relapse curve and long-term abstinence among untreated smokers. Addiction 2004 Jan;99(1):29-38. [doi: 10.1111/j.1360-0443.2004.00540.x] [Medline: 14678060]

3. Ekelund U, Steene-Johannessen J, Brown WJ, Fagerland MW, Owen N, Powell KE, Lancet Physical Activity Series 2 Executive Committe, Lancet Sedentary Behaviour Working Group. Does physical activity attenuate, or even eliminate, the detrimental association of sitting time with mortality? A harmonised meta-analysis of data from more than 1 million men and women. Lancet 2016 Sep 24;388(10051):1302-1310. [doi: 10.1016/S0140-6736(16)30370-1] [Medline: 27475271]

4. Office of Disease Prevention and Health Promotion. 2018. 2018 Physical Activity Guidelines Advisory Committee Scientific Report URL: https://health.gov/paguidelines/second-edition/report/pdf/PAG_Advisory_Committee_Report.pdf [accessed 2019-01-29]

5. Walker A, Langdon J, Johnson K. Relationships among meeting physical-activity guidelines and health risk behaviors. J Phys Act Health 2015 Jun;12(6):776-781. [doi: 10.1123/jpah.2014-0079] [Medline: 25110985]

6. Milicic S, Piérard E, DeCicca P, Leatherdale ST. Examining the association between physical activity, sedentary behavior and sport participation with e-cigarette use and smoking status in a large sample of Canadian youth. Nicotine Tob Res 2019 Feb 18;21(3):285-292 [FREE Full text] [doi: 10.1093/ntr/ntx238] [Medline: 29099946]

7. Ussher MH, Taylor AH, Faulkner GE. Exercise interventions for smoking cessation. Cochrane Database Syst Rev 2014 Aug 29(8):CD002295. [doi: 10.1002/14651858.CD002295.pub5] [Medline: 25170798]

8. Thompson TP, Greaves CJ, Ayres R, Aveyard P, Warren FC, Byng R, et al. An exploratory analysis of the smoking and physical activity outcomes from a pilot randomized controlled trial of an exercise assisted reduction to stop smoking intervention in disadvantaged groups. Nicotine Tob Res 2016 Mar;18(3):289-297. [doi: 10.1093/ntr/ntv099] [Medline: 25969453]

9. Klinsophon T, Thaveeratitham P, Sitthipornvorakul E, Janwantanakul P. Effect of exercise type on smoking cessation: a meta-analysis of randomized controlled trials. BMC Res Notes 2017 Sep 6;10(1):442 [FREE Full text] [doi: 10.1186/s13104-017-2762-y] [Medline: 28874175] 
10. Haasova M, Warren F, Ussher M, van Rensburg KJ, Faulkner G, Cropley M, et al. The acute effects of physical activity on cigarette cravings: systematic review and meta-analysis with individual participant data. Addiction 2013 Jan;108(1):26-37. [doi: 10.1111/j.1360-0443.2012.04034.x] [Medline: 22861822]

11. Haasova M, Warren FC, Ussher M, van Rensburg KJ, Faulkner G, Cropley M, et al. The acute effects of physical activity on cigarette cravings: exploration of potential moderators, mediators and physical activity attributes using individual participant data (IPD) meta-analyses. Psychopharmacology (Berl) 2014 Apr;231(7):1267-1275. [doi: 10.1007/s00213-014-3450-4] [Medline: 24522330]

12. Haasova M, Warren FC, Thompson T, Ussher M, Taylor AH. The association between habitual physical activity and cigarette cravings, and influence of smokers' characteristics in disadvantaged smokers not ready to quit. Psychopharmacology (Berl) 2016 Jul;233(14):2765-2774 [FREE Full text] [doi: 10.1007/s00213-016-4326-6] [Medline: 27256353]

13. Knell G, Gabriel K, Businelle M, Shuval K, Wetter D, Kendzor D. Ecological momentary assessment of physical activity: validation study. J Med Internet Res 2017 Jul 18;19(7):e253 [FREE Full text] [doi: 10.2196/jmir.7602] [Medline: 28720556]

14. Silverman-Lloyd LG, Kianoush S, Blaha MJ, Sabina AB, Graham GN, Martin SS. mActive-Smoke: a prospective observational study using mobile health tools to assess the association of physical activity with smoking urges. JMIR Mhealth Uhealth 2018 May 11;6(5):e121 [FREE Full text] [doi: 10.2196/mhealth.9292] [Medline: 29752250]

15. Craig CL, Marshall AL, Sjöström M, Bauman AE, Booth ML, Ainsworth BE, et al. International physical activity questionnaire: 12-country reliability and validity. Med Sci Sports Exerc 2003 Aug;35(8):1381-1395. [doi: 10.1249/01.MSS.0000078924.61453.FB] [Medline: 12900694]

16. Bauman A, Bull F, Chey T, Craig C, Ainsworth B, Sallis J, et al. The International Prevalence Study on Physical Activity: results from 20 countries. Int J Behav Nutr Phys Act 2009 Mar 31;6:21 [FREE Full text] [doi: 10.1186/1479-5868-6-21] [Medline: 19335883 ]

17. The University of Arizona Cancer Center. Behavior Measurement and Interventions URL: https://cancercenter.arizona.edu/ researchers/shared-resources/behavioral-measurement-and-interventions [accessed 2019-06-05] [WebCite Cache ID $78 \mathrm{uCl}$ Pho]

18. Fitabase. 2018. Fitabase Data Dictionary URL: https://www.fitabase.com/media/1748/fitabasedatadictionary.pdf [accessed 2019-06-06] [WebCite Cache ID 78w1eBN4d]

19. Schrack JA, Cooper R, Koster A, Shiroma EJ, Murabito JM, Rejeski WJ, et al. Assessing daily physical activity in older adults: unraveling the complexity of monitors, measures, and methods. J Gerontol A Biol Sci Med Sci 2016 Aug;71(8):1039-1048 [FREE Full text] [doi: 10.1093/gerona/glw026] [Medline: 26957472]

20. Tucker JM, Welk GJ, Beyler NK. Physical activity in US: adults compliance with the Physical Activity Guidelines for Americans. Am J Prev Med 2011 Apr;40(4):454-461. [doi: 10.1016/j.amepre.2010.12.016] [Medline: 21406280]

21. Dominick GM, Winfree KN, Pohlig RT, Papas MA. Physical activity assessment between consumer- and research-grade accelerometers: a comparative study in free-living conditions. JMIR Mhealth Uhealth 2016 Sep 19;4(3):e110 [FREE Full text] [doi: 10.2196/mhealth.6281] [Medline: 27644334]

22. Evenson KR, Goto MM, Furberg RD. Systematic review of the validity and reliability of consumer-wearable activity trackers. Int J Behav Nutr Phys Act 2015 Dec 18;12:159 [FREE Full text] [doi: 10.1186/s12966-015-0314-1] [Medline: 26684758]

23. Wang R, Blackburn G, Desai M, Phelan D, Gillinov L, Houghtaling P, et al. Accuracy of wrist-worn heart rate monitors. JAMA Cardiol 2017 Jan 1;2(1):104-106. [doi: 10.1001/jamacardio.2016.3340] [Medline: 27732703]

24. Tudor-Locke C, Han H, Aguiar E, Barreira TV, Schuna JM, Kang M, et al. How fast is fast enough? Walking cadence (steps/min) as a practical estimate of intensity in adults: a narrative review. Br J Sports Med 2018 Jun;52(12):776-788 [FREE Full text] [doi: 10.1136/bjsports-2017-097628] [Medline: 29858465]

25. Tudor-Locke C, Craig CL, Brown WJ, Clemes SA, de Cocker K, Giles-Corti B, et al. How many steps/day are enough? For adults. Int J Behav Nutr Phys Act 2011 Jul 28;8:79 [FREE Full text] [doi: 10.1186/1479-5868-8-79] [Medline: 21798015]

26. Tudor-Locke C, Camhi SM, Leonardi C, Johnson WD, Katzmarzyk PT, Earnest CP, et al. Patterns of adult stepping cadence in the 2005-2006 NHANES. Prev Med 2011 Sep;53(3):178-181. [doi: 10.1016/j.ypmed.2011.06.004] [Medline: 21708187]

27. Alharbi M, Bauman A, Neubeck L, Gallagher R. Validation of Fitbit-Flex as a measure of free-living physical activity in a community-based phase III cardiac rehabilitation population. Eur J Prev Cardiol 2016 Sep;23(14):1476-1485. [doi: 10.1177/2047487316634883] [Medline: 26907794]

\section{Abbreviations}

IPAQ: International Physical Activity Questionnaire

MET: metabolic equivalent of task

mHealth: mobile health

MVPA: moderate-to-vigorous physical activity

NHANES: National Health and Nutrition Examination Survey

VPA: vigorous physical activity 
Edited by N Bruining; submitted 11.06.19; peer-reviewed by G Knell, M Andrews, K Winfree; comments to author 14.08.19; revised version received 30.09.19; accepted 01.11.19; published 06.01.20

Please cite as:

Shan R, Yanek LR, Silverman-Lloyd LG, Kianoush S, Blaha MJ, German CA, Graham GN, Martin SS

Using Mobile Health Tools to Assess Physical Activity Guideline Adherence and Smoking Urges: Secondary Analysis of mActive-Smoke JMIR Cardio 2020;4(1):e14963

URL: https://cardio.jmir.org/2020/1/e14963

doi: $10.2196 / 14963$

PMID: $\underline{31904575}$

CRongzi Shan, Lisa R Yanek, Luke G Silverman-Lloyd, Sina Kianoush, Michael J Blaha, Charles A German, Garth N Graham, Seth S Martin. Originally published in JMIR Cardio (http://cardio.jmir.org), 06.01.2020. This is an open-access article distributed under the terms of the Creative Commons Attribution License (https://creativecommons.org/licenses/by/4.0/), which permits unrestricted use, distribution, and reproduction in any medium, provided the original work, first published in JMIR Cardio, is properly cited. The complete bibliographic information, a link to the original publication on http://cardio.jmir.org, as well as this copyright and license information must be included. 